\title{
Green und Sustainable Software im Kontext von Software Qualitätsmodellen
}

\author{
Julia Gerstlacher $\mathbb{D}$ - Iris Groher (D) Reinhold Plösch $(\mathbb{D}$
}

Eingegangen: 15. April 2021 / Angenommen: 18. November 2021 / Online publiziert: 20. Dezember 2021 (C) Der/die Autor(en) 2021

Zusammenfassung Insbesondere durch die gegenwärtige Corona-Krise ist der Digitalisierungsdruck gestiegen. Doch Digitalisierung hat auch ihre Schattenseiten, in Form von steigenden Emissionen und einem hohen Bedarf an Energie und Ressourcen. Konzepte wie Green Software, Green in IT und Green by IT sollen ein Bewusstsein für diese Thematik schaffen und dazu beitragen Nachhaltigkeitsziele zu erreichen. Ziel dieser Publikation ist die Untersuchung von Green Software Qualitätsmodellen, welche auf dem Standard ISO/IEC 25010 basieren. Im Rahmen einer Literaturrecherche haben wir Publikationen herausgefiltert, welche im Zeitraum 2011 bis 2020 veröffentlicht wurden und sich mit Green Software und Nachhaltigkeit im Kontext von Softwarequalität (ISO/IEC 25010) beschäftigt haben. Wir haben 7 verschiedene Modelle gefunden, welche Nachhaltigkeit in der folgenden Weise berücksichtigen: als eigenes Konzept von Softwarequalität (2 von 7), als Erweiterung des ISO/IEC 25010 (3 von 7), Nachhaltigkeit im ISO/IEC 25010 bereits enthalten (1 von 7), als Einflussfaktor, aber nicht als Teil der Produktqualität. Ein Modell lässt sich zu mehreren Betrachtungsweisen zuordnen. Fast alle Modelle (5 von 7) adressieren dabei die Dimension der ökologischen Nachhaltigkeit. Soziale Nachhal-

\footnotetext{
Julia Gerstlacher

Traun, Österreich

Johannes Kepler Universität Linz, Linz, Österreich

E-Mail: j.gerstlacher@gmx.de

Iris Groher · Reinhold Plösch

Institut für Wirtschaftsinformatik - Software Engineering, Johannes Kepler Universität Linz, Linz,

Österreich

Iris Groher

E-Mail: iris.groher@jku.at

Reinhold Plösch

E-Mail: reinhold.ploesch@jku.at
} 
tigkeit wird in 2 von 7 Modellen angesprochen. Lediglich 1 Modell berücksichtigt zusätzlich ökonomische, technische und individuelle Nachhaltigkeit. Im Kontext von Green und Sustainable Software werden, in den 7 Modellen, die folgenden Kriterien des ISO/IEC 25010 am häufigsten genannt: Performance Efficiency, Usability und Freedom from risk. Unser Beitrag liegt in der Identifikation von Green Software Qualitätsmodellen und dem ausführlichen Vergleich der einzelnen Modelle. Insbesondere analysieren wir, welche Qualitätskriterien des ISO/IEC 25010 als relevant für die Entwicklung von Green und Sustainable Software angesehen werden und wie die einzelnen Modelle Nachhaltigkeit adressieren.

Schlüsselwörter Green und Sustainable Software - Nachhaltigkeit und Software • Softwareproduktqualität · Software Qualitätsmodelle · ISO/IEC 25010

\title{
Green and Sustainable Software in the Context of Software Quality Models
}

\begin{abstract}
The current Corona crisis is a driving force for digitisation. Concepts such as Green Software, Green in IT and Green by IT should create awareness for the downsides of digitisation, like a high demand for energy and resources, and contribute to achieving sustainability goals. This article aims at investigating Green Software Quality Models based on the ISO/IEC 25010 standard. By means of a literature search, we identified publications in the period from 2011 to 2020 that deal with the topics of Green Software and Sustainability in the context of Software Quality (ISO/IEC 25010). We found 7 different models that consider Sustainability in the following way: as a separate concept of Software Quality (2 of 7), as an extension of ISO/IEC 25010 (3 of 7), Sustainability already included in ISO/IEC 25010 (1 of 7), as an influencing factor but not as part of quality. One model can be assigned to more than one point of view. Almost all models (5 out of 7) address the dimension of Environmental Sustainability. Social Sustainability is addressed in 2 out of 7 models. Economic, Technical, and Individual Sustainability rank third (1 out of 7). In the context of Green and Sustainable Software, the following criteria of ISO/IEC 25010 are mentioned most frequently: Performance Efficiency, Usability, and Freedom from risk. Our contribution lies in the identification of Green Software Quality Models and the detailed comparison of the individual models. We analyse which criteria of the ISO/IEC 25010 standard are considered relevant for developing Green and Sustainable Software and how the models address Sustainability.
\end{abstract}

Keywords Green and Sustainable Software - Software Product Quality · Software Quality Model · ISO/IEC 25010

\section{Motivation und Einleitung}

Im Rahmen der „Agenda 2030 für nachhaltige Entwicklung“ (Nachhaltige Entwicklung - Agenda 2030/SDGs - Bundeskanzleramt Österreich, o.J.; Sustainable Development Goals o.J.) haben sich die 193 Mitgliedsstaaten der Vereinten Nationen 
(UN) auf insgesamt 17 Entwicklungsziele, die sogenannten Sustainable Development Goals (SDGs), geeinigt deren Erfüllung spätestens im Jahr 2030 abgeschlossen sein soll. Die SDGs umfassen die Dimensionen Wirtschaft, Soziales und Ökologie.

Bei der Umsetzung der SDGs kann Digitalisierung helfen, und zwar in Form von sogenannter Green Software, Green in IT oder Green by IT. Green in IT liegt dann vor, wenn das Softwareprodukt selbst einen möglichst geringen negativen oder gar einen positiven Einfluss auf Umwelt und Ressourcen hat (Naumann et al. 2011, 2015). Wenn mithilfe eines Softwareproduktes Nachhaltigkeitsziele erreicht werden können, spricht man von Green by IT (Condori-Fernandez und Lago 2019, S. 1). In dieser Arbeit konzentrieren wir uns auf den Aspekt Green in IT und betrachten Nachhaltigkeit im Green Kontext.

Qualitätsmodelle helfen dabei Anforderungen an Software zu formulieren, zu messen und zu beurteilen (Kläs et al. 2009). Ein wesentliches Ziel dieser Arbeit ist die Beantwortung der Frage, inwieweit im Bereich der Nachhaltigkeit bereits qualitätsmodellorientierte Ansätze existieren und ob diese Ansätze eine tragfähige Basis für die Entwicklung eines operativen Qualitätsmodells für Green und Sustainable Software darstellen. Im Rahmen der vorgenannten Forschungsfrage untersuchen wir, welche grünen Modelle es gibt, die auf dem ISO/IEC-Standard 25010 zur Softwareprodukt- und Nutzungsqualität (ISO/IEC 25010 2011) basieren. Die Beschränkung auf den ISO/IEC-Standard 25010 nehmen wir vor, weil dieser weltweit gesehen der wichtigste Standard im Bereich der Softwarequalität ist. Damit betrachten wir in erster Linie den Aspekt von Green Software mit dem Verständnis, durch Green in IT (s. oben) einen positiven Nachhaltigkeitseffekt zu erzielen.

Die gefundenen Modelle vergleichen wir dahingehend, wie sie Nachhaltigkeitsdimensionen berücksichtigen, welche Kriterien des ISO/IEC 25010 sie im Kontext von Green Software aufgreifen und in welchem Zusammenhang sie mit der ISONorm stehen. Wir betrachten bei unserer Analyse Publikationen der Jahre 2011 bis 2020, da das Thema Green und Sustainable Software seit 2011 zunehmend im Interesse der ForscherInnen steht (Calero et al. 2020; García-Mireles et al. 2018). Ziel dieser Arbeit ist demnach herauszufinden, inwieweit sich Qualitätsattribute herauskristallisiert haben, die PraktikerInnen bei der Entscheidung darüber helfen, welche Qualitätsaspekte im Fokus stehen sollten, um Nachhaltigkeitsziele zu adressieren.

Abschn. 2 gibt einen Überblick über Arbeiten auf dem Gebiet Green und Sustainable Software. Die im Rahmen dieser Arbeit angewandte Forschungsmethodik wird in Abschn. 3 vorgestellt. Abschn. 4 präsentiert die identifizierten Qualitätsmodelle und deren Bezug zu Green und Sustainability. Ein zusammenfassender Vergleich der Modelle findet sich in Abschn. 5. Der Artikel endet mit einer Diskussion und einem Ausblick auf mögliche zukünftige Entwicklungen auf dem Gebiet grüner Softwarequalitätsmodelle.

\section{Green und Sustainable Software}

Einen Überblick über das Thema Green und Sustainable Software und dessen Entwicklung seit den Anfängen im Jahr 2000, haben sich Calero et al. (2020) im Jahr 2018 verschafft. Ihre Ergebnisse haben sie in der Publikation „5 Ws of Green and 
Sustainable Software“ (Calero et al. 2020) veröffentlicht, welche im Jahr 2020 erschienen ist.

Die von Calero et al. durchgeführte Analyse wurde auf Basis der bibliographischen Datenbank SCOPUS durchgeführt. Um die Schlüsselkategorien des Forschungsgebiets Green und Sustainable Software aus der Literatur zu extrahieren, haben Calero et al. (2020) nach englischen Publikationen gesucht, die im Zeitraum von 2000 bis Anfang November 2018 veröffentlicht wurden. Insgesamt haben sie 542 Studien gefunden, deren Veröffentlichungsdatum größtenteils ab 2011 war. Den größten Einfluss auf die Forschung zu Green und Sustainable Software hatten die Forscherteams rund um die Autorinnen Lago, Penzenstadler, Calero, Kern und den Autor Hindle. Bezugnehmend auf den Software Engineering Body of Knowledge (SWEBOK o.J.) hat die meiste Forschung dabei im Bereich der Softwarequalität stattgefunden (vgl. Calero et al. 2020, S. 411). Im Zusammenhang mit nachhaltigen Aspekten werden laut Calero et al. (2020) die folgenden Schlagworte am häufigsten genannt: Green Software, Green IT, Software Sustainability, Energy Consumption und Energy Efficiency. Die AutorInnen kommen zu dem Ergebnis, dass das Forschungsgebiet rund um Green und Sustainable Software mittlerweile eine gute Reife erlangt hat und die Forschung aktiv und multinational betrieben wird.

\section{Vorgehensweise}

Der Fokus dieser Arbeit liegt auf der Untersuchung von Softwarequalitätsmodellen, welche nachhaltige bzw. grüne Aspekte berücksichtigen, auf dem Standard ISO/IEC 25010 basieren und im Zeitraum von 2011 bis 2020 veröffentlicht wurden. Betrachtet werden einerseits Modelle, welche die strukturelle Integrität des Standards wahren und andererseits Modelle, die beispielsweise einzelne Kriterien des Standards adressieren. Modelle, welche ausschließlich auf der Norm ISO/IEC 9126 basieren, sind nicht Teil dieses Artikels.

Ausgangspunkt unserer Literaturstudie war die Publikation „Green Software and Software Quality“ von Calero et al. (2015), die im Buch „Green in Software Engineering“ erschienen ist. Schwerpunkt dieser Publikation war die Analyse, wie grüne bzw. ökologische Aspekte bei der Entwicklung von Softwareprodukten berücksichtigt werden könnten. Als Green Software oder Software Greenability bezeichnen Calero et al. (vgl. 2015, S. 232) dabei jene Software, bei der im Entwicklungsprozess ein ökologisch nachhaltiger Einsatz von Ressourcen gleichbedeutend mit der Erfüllung der Anforderungen an die Software/das Softwareprodukt ist. Im Rahmen ihrer Forschungstätigkeit haben Calero et al. den Standard ISO/IEC 25010:2010 in den Ausprägungen Softwareproduktqualität (Product Quality, PQ) und Nutzungsqualität (Quality in Use, QiU) dahingehend analysiert, inwiefern dieser bereits nachhaltige Aspekte enthält. Die AutorInnen haben hierfür die PQ-Dimension Performance Efficiency mit den eigens definierten Charakteristika für Greenability verglichen, mit dem Ergebnis, dass der ISO-Standard um die Dimension Greenability erweitert werden muss, um den Ressourcenverbrauch aus Performance- und aus Umweltperspektive betrachten zu können. 
Tab. 1 Suchwörter

\begin{tabular}{ll}
\hline Stichwörter & Synonyme \\
\hline $\begin{array}{l}\text { Green und Sus- } \\
\text { tainable Software } \\
\text { Software Product } \\
\begin{array}{l}\text { Quality (PQ) } \\
\text { Software Quality }\end{array}\end{array}$ & $\begin{array}{l}\text { Greener Software, Green IT, Green ICT, Environmental Sustainability, Sustainabil- } \\
\text { Quality, Product Quality, Software Quality, Green Software Quality } \\
\text { Model/s }\end{array}$ \\
& $\begin{array}{l}\text { Quality Modeling, Quality Model/s, Software Quality Modeling, IT-oriented Qua- } \\
\text { lity Model, ISO 25010, ISO/IEC 25010, ISO 9126, ISO/IEC 9126, Software Qua- }\end{array}$ \\
\hline
\end{tabular}

aSO 9126 bzw. ISO/IEC 9126 wurden als Suchwörter ausgewählt, da sie ein Softwarequalitätsmodell darstellen. Modelle, die ausschließlich auf ISO/IEC 9126 basieren, wurden in dieser Arbeit jedoch nicht betrachtet, da das ISO/IEC 9126 veraltet ist und durch das ISO/IEC 25010 ersetzt wurde (vgl. ISO/IEC 9126-1 o.J.)

Zur Beantwortung unserer Forschungsfrage wurde die Ausgangsliteratur „Green Software and Software Quality“ (Calero et al. 2015) nach relevanten Begriffen für die weitere Suche durchforstet. Ausgehend von diesem Artikel wurden die in Tab. 1 dargestellten Suchwörter abgeleitet.

Für die Suche nach Publikationen, welche von Green und Sustainable Software im Kontext von Softwarequalitätsmodellen handeln, wurden die obigen Suchwörter mit AND und/oder OR verknüpft. Zudem wurden die Literaturverzeichnisse der gefundenen Artikel nach weiteren, potenziell interessanten, sprich der Beantwortung der Fragestellung dienlichen Quellen durchsucht. Für die Literatursuche wurde die erweiterte Suchfunktion der wissenschaftlichen Datenbanken SpringerLink, ScienceDirect und SCOPUS genutzt. Darüber hinaus wurde das soziale Netzwerk Researchgate und Google Scholar durchsucht. Google Scholar wurde hierbei für die Breitensuche über die jeweiligen wissenschaftlichen Datenbanken, wie SpringerLink, ScienceDirect, ACM, IEEE Xplore verwendet. Gesucht wurde im Titel, Abstract und den Schlagwörtern der jeweiligen Publikationen. Die Suche fand im Zeitraum vom 08.10.2020 bis 31.12.2020 statt. Zudem wurden während der Ausarbeitung weitere Quellen gefunden, die ebenfalls ihre Berücksichtigung in dieser Publikation finden. Unsere Suche hat 34 Literaturquellen hervorgebracht ${ }^{1}$. Im nachfolgenden Kapitel stellen wir die identifizierten Weiterentwicklungen des ISO/IEC 25010 dar.

\section{Analyseergebnisse}

In diesem Kapitel stellen wir Qualitätsmodelle vor, die das Thema Sustainability, im Kontext von Green Software, betrachten sowie wissenschaftlich fundiert sind. Weiters beurteilen wir, inwieweit diese Modelle systematische Erweiterungen der ISO/IEC 25010 sind.

Basis für die Analyse der Qualitätsmodelle bildet das ISO/IEC 25010, welches zwei wesentliche Teilmodelle umfasst: das Softwarequalitätsmodell (Product Qua-

\footnotetext{
1 Siehe https://bit.ly/3DkRAu9.
} 
lity Model, PQ) und das Nutzungsqualitätsmodell (Quality in Use Model, QiU). Das PQ besteht aus den Qualitätsattributen Functional Suitability, Performance Efficiency, Compatibility, Usability, Reliability, Security, Maintainability und Portability. Das QiU wiederum konzentriert sich auf die Qualitätsattribute Effectiveness, Efficiency, Satisfaction, Freedom from risk und Context Coverage.

In den folgenden Absätzen werden die identifizierten grünen Softwarequalitätsmodelle kurz vorgestellt und für jedes Modell geprüft, inwieweit es einen Bezug zum ISO/IEC 25010 herstellt und Nachhaltigkeit berücksichtigt.

\subsection{Modell 1: Quality Model Green and Sustainable Software von Kern et al. (2013) bzw. Naumann et al. (2015)}

Naumann et al. (2011) haben im Jahr 2011 das GREENSOFT Modell präsentiert, welches mittels Betrachtung des gesamten Produktlebenszyklus die Nachhaltigkeitsdimensionen Ökologie, Soziales, Wirtschaft und Menschheit berücksichtigen soll (Naumann et al. 2011, S. 296).

Kern et al. (2013, S. 89) verorten eine Forschungslücke, wenn es darum geht, direkte (sogenannte Effekte erster Ordnung) und indirekte Auswirkungen (sogenannte Effekte zweiter und dritter Ordnung) von Software auf die Umwelt zu erfassen. Aus diesem Grund haben sie (Kern et al. 2013) das GREENSOFT Modell um das Submodell Quality Model for Green and Sustainable Software erweitert. Der Fokus ihres Teilmodells liegt auf der Definition von Kriterien und Metriken, um feststellen zu können, ob das zu analysierende Softwareprodukt dem Feld Green Software und Green Softwareengineering zuzuordnen ist. Es konkretisiert dabei die GREENSOFT-Dimension Sustainability Criteria and Metrics, die sich aus den drei Kategorien Common Quality Criteria and Metrics, Directly Related Criteria and Metrics und Indirectly Related Criteria and Metrics zusammensetzt. Darüber hinaus erlaubt es Effekte erster, zweiter und dritter Ordnung systematisch zu analysieren und bei der Entwicklung von Software zu berücksichtigen. Soziale Aspekte finden ihre Entsprechung in den Directly related Criteria des Quality Model for Green and Sustainable Software (Kern et al. 2013; Naumann et al. 2015). Ökologische Aspekte werden auf allen drei Ebenen des Modells berücksichtigt.

Die Norm ISO/IEC 25010 (2011) berücksichtigen die EntwicklerIn (Kern et al. 2013; Naumann et al. 2015) im Quality Model for Green and Sustainable Software indirekt, und zwar in der Form, dass sie die folgenden Kriterien des PQs in ihre Kategorien Common Criteria und Directly related Criteria aufnehmen:

- Resource utilization (Subkriterium von Performance Efficiency),

- Usability, mit Subkriterium Accessibility und

- Portability, mit Subkriterium Adaptability.

Darüber hinaus erachten Kern et al. (2013) die Kriterien Reusability und Modifiability (Subkriterien des PQ-Kriteriums Maintainablilty) als relevant für Sustainable Development. Einen darüberhinausgehenden Bezug zum ISO/IEC 25010 stellen sie nicht her. 


\subsection{Modell 2: 25010+S von Calero et al. (2015)}

Calero et al. (2014a, 2015) sind der Meinung, dass die ökologische Nachhaltigkeitsdimension in jedem Fall bei der Softwareproduktentwicklung berücksichtigt werden sollte, und bezeichnen dies als Green Software oder Software Greenability. Sie begründen dies durch den Ressourcenverbrauch, welcher durch die Produktion und den Betrieb von Software getrieben ist. In (Calero et al. 2015) stellen sie einen direkten Bezug zum ISO/IEC 25010 her, in dem sie zu Beginn untersuchen, ob die Norm Sustainability bereits implizit, in Form von Performance Efficiency, berücksichtigt. Sie kommen zu dem Ergebnis, dass das ISO/IEC 25010 um die Dimension Greenablity erweitert werden muss, um Nachhaltigkeit im Softwareentwicklungsprozess explizit berücksichtigen zu können. Konkret geht es ihnen um die Formulierung von grünen nichtfunktionalen Anforderungen bzw. Greenability-Kriterien. Als Folge haben sie das PQ-Modell und QiU-Modell des Standards um eine neue Dimension Greenability erweitert und hierfür entsprechende Subkriterien festgelegt. Im Kontext von Green Software und Softwareproduktqualität (PQ) erachten sie folgende Kriterien als relevant:

- Greenability als Erweiterung des PQ-Modells

- Energy efficiency

- Resource ${ }^{2}$ optimisation

- Capacity optimisation

- Perdurability

Damit ein Softwareprodukt langlebig ist, braucht es nach Ansicht von Calero et al. (2015, S. 240) das Subkriterium Perdurability, welches von den Subkriterien Modifiability, Adaptability und Reusability abgeleitet ist.

Auch beim QiU-Modell haben Calero et al. überprüft, ob Greenability bereits implizit berücksichtigt ist. Sie beschreiben dies vage als „,identifying those characteristics and sub-characteristics that may be related in some way to greenability“ (Calero et al. 2015, S. 243). Eine Aufzählung, welche (Sub-)Kriterien dies sein könnten fehlt ${ }^{3}$. Im Ergebnis erweitern Calero et al. (2015, S. 245) das QiU-Modell des ISO/IEC 25010 wie folgt:

- Greenability als Erweiterung des QiU-Modells

- Efficiency optimisation

- User's environmental perception

- Minimisation of environmental effects

\footnotetext{
2 Als Ressourcen erachten Calero et al. (2015, S. 238) jene, die im ISO-Standard (ISO/IEC 25010 2011, S. 11) genannt werden, also exklusive Humanressourcen.

${ }^{3}$ Auch in (Calero et al. 2014a; Calero et al. 2013a) wird die Vorgehensweise nicht näher beschrieben.
} 


\subsection{Modell 3: Generic Sustainable Software Star Model (GS3M) von Amri und Bellamine Ben Saoud (2014a, b)}

Den Forschenden Amri und Bellamine Ben Saoud (2014a) gingen die bisherigen Bestrebungen, wie Nachhaltigkeit im Software Engineering Berücksichtigung finden könnte, nicht weit genug, da AutorInnen wie beispielsweise Kern et al. (2013) oder Calero et al. (2013b, 2014b) lediglich die soziale und/oder die ökologische Nachhaltigkeitsdimension adressiert haben. Amri und Bellamine Ben Saoud (2014a) beziehen sich hierbei auf Penzenstadler und Femmer (2013), welche der Ansicht sind, dass nachhaltiges Software Engineering ökologisch, technisch, sozial, individuell ${ }^{4}$ und ökonomisch nachhaltig sein sollte.

Amri und Bellamine Ben Saoud (2014a, b) entwickelten daher das Generic Sustainable Software Star Model (GS3M), bei welchem jede Sternzacke eine der vorgenannten fünf Nachhaltigkeitsdimension adressiert. Im Fokus dieses Modells steht sowohl das Softwareprodukt als auch die zugrundeliegenden Prozesse. Auf die Norm ISO/IEC 25010 (2011) beziehen sich Amri und Bellamine Ben Saoud (2014a, b) insoweit, als dass sie ihre generalisierten Attribute durch Qualitätskriterien aus der Norm konkretisieren. Als Beispiel führen sie Accessibility an, welchem sie einen sozialen Mehrwert für AnwenderInnen eines Softwareprodukts attestieren. In Form von Green Software beziehen sie sich auf die ISO-Kriterien Performance Efficiency und indirekt auch auf Resource utilization. Als Voraussetzungen für die Dauerhaftigkeit von Software sehen sie die Kriterien Maintainability, Portability und Usability an.

\subsection{Modell 4: Green internal/external quality model von Beghoura et al. (2017)}

Einen ähnlichen Ansatz, wie jenen von Calero et al. (2015), haben Beghoura et al. (2017) in ihrer Publikation „Green software requirements and measurement: random decision forests-based software energy consumption profiling“ verfolgt. Beghoura et al. (2017) entwickelten einerseits das Green internal/external quality model und präsentierten andererseits ein Profiling-Tool, mit welchem sich die Softwarequalität und Green Efficiency von Software beurteilen lässt. Das Profiling-Tool basiert auf einem multiplen Regressionsmodell und verwendet einen überwachten Lernalgorithmus, um das Energieverbrauchsverhalten verschiedener Geräte, auf denen die Software unter verschiedenen Arbeitslastbedingungen läuft, zu untersuchen. Bezüglich weiterer Details zum Profiling-Tool wird auf (Beghoura et al. 2017, S. 30-39) verwiesen.

Beghoura et al. (2017, S. 29) heben weiters hervor, dass der Energieverbrauch von der Intensität der Ressourcennutzung und den zugrundeliegenden Hardwarekomponenten beeinflusst wird. Sie sind deshalb der Meinung, dass EntwicklerInnen Techniken anwenden sollten, die zur Optimierung des Energiebedarfs beitragen. Um

\footnotetext{
4 Unter individueller Nachhaltigkeit verstehen Amri und Bellamine Ben Saoud (2014a), dass der Prozess der Softwareentwicklung von den EntwicklerInnen, mit ihren Kenntnissen und Fähigkeiten, im Rahmen von angemessenen Arbeitszeiten, adäquater Bezahlung und fairen Arbeitsbedingungen umgesetzt werden kann.
} 
eine differenzierte Betrachtung, je nach Art der zu entwickelten Software und der benötigten Hardwarekomponenten, zu ermöglichen, haben die Autoren (Beghoura et al. 2017, S. 29) die folgenden Kriterien definiert:

- Green computation efficiency ist dann gegeben, wenn die Software die geforderte Arbeitslast effizient und mit optimalen Energieeinsatz bewältigen kann.

- Mittels Green data management soll gewährleistet werden, dass die Software auf gespeicherte Daten möglichst effizient und energiesparend zugreifen kann.

- Bei Green data communication liegt der Fokus auf Energiemanagementstrategien, die einen möglichst energieeffizienten Datenaustausch über das Netzwerk ermöglichen sollen.

- Eine ganzheitliche Betrachtung des Energieverbrauchs von Software kann mittels Energy consumption awareness erreicht werden. Ziel hierbei ist es, ein Bewusstsein für das Verhalten von Software bei hohem, durchschnittlichen und niedrigem Energieverbrauch zu erlangen.

Bezugnehmend auf das QiU-Modell, des Standards ISO/IEC 25010 (2011, S. 3), kommen Beghoura et al. (2017, S. 29) zu dem Schluss, dass grüne Softwarequalitätsaspekte bereits implizit, in Form des Kriteriums Freedom from risk, berücksichtigt sind. Die Autoren bemängeln jedoch, dass das ISO-Modell für Produktqualität (PQ) (ISO/IEC 25010 2011, S. 4) keine Kriterien für die Entwicklung von Green Software bereitstellt.

Bezugnehmend auf die Dimensionen der SDGs, bieten Beghoura et al. (2017) mit ihrem Modell die Möglichkeit nachhaltige Anforderungen an Software zu definieren und so ökologische Nachhaltigkeit im Softwareentwicklungsprozess zu adressieren.

\subsection{Modell 5: Integrating Environmental Sustainability in Software Product Quality von Koçak et al. (2015, 2014)}

Koçak et al. haben evaluiert (2013, 2014, 2015), wie sich Anforderungen an die Softwarequalität und an die Umweltfreundlichkeit des Produktes wechselseitig beeinflussen. Sie haben zudem ein hierarchisches Rahmenwerk präsentiert (Koçak et al. 2013, 2014), mit welchem Green Software Kriterien priorisiert werden können, um Zielkonflikte auszugleichen bzw. zu minimieren.

In (Koçak et al. 2015, S. 2-3) beziehen sie sich auf die Qualitätsmodelle des ISO/IEC 9126 und des ISO/IEC 25010 und heben hervor, dass Nachhaltigkeit in diesen keine Berücksichtigung findet. Als Modelle, welche Green und Sustainable Software berücksichtigen und die Norm ISO/IEC 25010 referenzieren, wählten Koçak et al. (2015, S. 3) das Softwarequalitätsmodell von Kern et al. (2013) sowie das Modell 25010+S von Calero et al. $(2013 \mathrm{a}, 2015)$ aus. In diesem Zusammenhang identifizierten sie die Erforschung des Einflusses von Qualität auf Nachhaltigkeitsdimensionen als Forschungslücke. Um diese Forschungslücke zu schließen, überführten Koçak et al. (2015) zunächst die Hierarchiestufen aus (Koçak et al. 2014) in ein Modell. Auf Ebene der Softwarequalität erachten Koçak et al. (2015), die folgenden internen Qualitätskriterien des PQ-Modells (ISO/IEC 25010 2011) als relevant: Functional Suitability, Performance Efficiency, Security, Reliability, Usability und Compatibility. Die anschließende Befragung von 53 IT-ExpertInnen hat 
ergeben, dass lediglich Performance Efficiency, Usability, Functional Suitability und Reliability als wichtige Kriterien für die Entwicklung von ökologisch nachhaltigen Softwareprodukten angesehen werden (Koçak et al. 2015, S. 7).

Für die Environmental-Dimension haben Koçak et al. Resource usage und Energy impact (aus Koçak et al. 2014) in Resource Efficiency und Energy Efficiency umbenannt. Die Autorinnen (Koçak et al. 2015) begründen ihre Auswahl damit, dass diese Kriterien mit einem direkten Effekt auf Umwelt oder Ressourcen assoziiert werden und daher im Fokus ihrer Untersuchung stehen.

Wenn die benötigten Ressourcen, welche das Softwareprodukt für die Ausführung von Funktionen oder dem Produzieren nützlicher Arbeitslast nutzt, effizient arbeiten, dann sprechen Koçak et al. (2015, S. 4) von Ressource Efficiency. Als Beispiele für datenverarbeitende Ressourcen nennen sie unter anderem Arbeitsspeicher, Netzwerkbandbreite oder Storage (Koçak et al. 2015, S. 4).

\subsection{Modell 6: IT-oriented Software Quality Models von Gordieiev und Kharchenko (2018)}

Gordieiev et al. (2015, 2016) haben in den Jahren 2015 und 2016 bestehende Softwarequalitätsmodelle auf deren Entwicklung im Zusammenhang mit Green Software und Software Reliability untersucht und eine Vorhersage getroffen, wie die zweite Version der Norm ISO/IEC 25010 (PQ- und QiU-Modell) Aspekte von Green Software berücksichtigen könnte. Öffentlich zugängliche Informationen zum Inhalt des überarbeiteten Standards (ISO/IEC CD 25010 o.J.) sind nicht verfügbar.

Darüber hinaus haben sie im Jahr 2018 den Artikel „IT-oriented software quality models and evolution of the prevailing characteristics" (Gordieiev und Kharchenko 2018) veröffentlicht. Die Autoren haben in diesem wechselseitige Beziehungen zwischen den Kriterien des ISO/IEC 25010 und Informationstechnologien (IT) sowie Paradigmen der Informatik untersucht. Konkret wurde die Korrelation für die Begriffe Internet of Things (IoT), Green IT, Virtual Reality (VR), Augmented Reality $(A R)$, Artificial Intelligence (AI), Cloud Computing, Blockchain und Web betrachtet. Weiters haben sie ein technologieorientiertes Softwarequalitätsmodell skizziert.

Für die Erstellung des IT-orientierten Softwarequalitätsmodells (ITOSQM) haben Gordieiev und Kharchenko (2018, S. 376) zunächst das grobe Konzept erstellt und sich dabei für das ISO/IEC 25010 als Basis entschieden und festgelegt, dass jedes ITOSQM beliebig viele Kriterien aus dem Standard enthalten darf. Die Kriterien selbst können als Typ 1, Typ 2 oder Typ 3 klassifiziert werden. Gordieiev und Kharchenko (2018, S. 376) beschreiben die Kategorien wie folgt:

1. Typ 1: Prioritätsmerkmale, die von der Informationstechnologie beeinflusst werden

2. Typ 2: wichtige Merkmale, die im Zusammenhang mit der Informationstechnologie stehen, aber nicht von höchster Priorität sind

3. Typ 3: Merkmale, die nicht im Zusammenhang mit der Informationstechnologie stehen 
Für die Erstellung des Modells haben die Autoren (Gordieiev und Kharchenko 2018) anschließend und mittels Fragebogen erhoben, wie ExpertInnen die $P Q$ Hauptkriterien des ISO/IEC 25010 (2011) den einzelnen Technologien und Konzepten zuordnen würden. Die ExpertInnen kamen dabei aus der Softwareentwicklung (30\%), dem IT-Sektor (20\%) und aus Forschungs- und Bildungseinrichtungen.

Die von Gordieiev und Kharchenko (2018) durchgeführte Befragung zur Erhebung von Beziehungen zwischen Softwarequalitätskriterien des ISO/IEC 25010 (Hauptkriterien des PQ-Modells) und IT Paradigmen hat ergeben, dass Performance Efficiency als prioritäres Merkmal zu sehen ist, welches von Green IT beeinflusst wird (Typ 1, 7 Zuordnungen). Zudem werden Functional suitability und Reliability (jeweils 4 Zuordnungen) sowie Usability (3 Zuordnungen) von den ExpertInnen als wichtig, aber nicht als vorranging (Typ 2) angesehen. Keine Beziehung zu Green IT (Typ 3) wurde den Kriterien Security und Portability (jeweils 2 Zuordnungen) sowie Compatibility und Maintainability (jeweils 1 Zuordnung) attestiert.

\subsection{Modell 7: Software Sustainability Model von Condori-Fernandez und Lago (2018a, b, 2019)}

Einen weiteren und ersten Ansatz, wie Nachhaltigkeit als Qualitätsanforderung im Softwareentwicklungsprozess berücksichtigt werden kann, haben Condori-Fernandez und Lago (2018a) in ihrer Publikation „Characterizing the contribution of quality requirements to software sustainability“ veröffentlicht. Sie haben dabei empirisch untersucht, welche Qualitätsanforderungen des ISO/IEC 25010 in der Lage sind zu ökonomischer, technischer, ökologischer und sozialer Nachhaltigkeit beizutragen. Konkret haben die Autorinnen mittels Fragebogen erhoben, welche Kriterien des ISO/IEC 25010 (Product Quality und Quality in Use, PQ und QiU) als relevant für die einzelnen Dimensionen von Nachhaltigkeit angesehen werden. Auf Basis der in (2018a) gewonnenen Erkenntnisse haben Condori-Fernandez und Lago in (2018b) das Software Sustainability Quality Model entwickelt, welches aus einer tabellarischen Zuordnung der ISO-Qualitätskriterien zu den Dimensionen sozialer, technischer, ökologischer und ökonomischer Nachhaltigkeit besteht. Im Zuge weiterer Publikationen (Condori-Fernandez und Lago 2019; Fernandez et al. 2019; Condori-Fernandez et al. 2020) haben die AutorInnen den Nutzen ihres Modells evaluiert und dieses sukzessive verfeinert.

Software ist per Definition dann Green, wenn sie einen möglichst geringen negativen und im besten Fall positiven Einfluss auf die Nachhaltigkeitsdimensionen hat (vgl. Naumann et al. 2011). Für die Entwicklung von Green und Sustainable Software sind dabei jene Kriterien des Software Sustainability Quality Modells besonders relevant, die mehrere Nachhaltigkeitsdimensionen adressieren. Quintessenz aller vorgenannten Publikationen ist, dass insbesondere die folgenden Kriterien des ISO/IEC 25010 als relevant für die Entwicklung von Green Software angesehen werden:

- Compatibility: technische, soziale, ökologische und ökonomische Nachhaltigkeit

- Effectiveness: technische, soziale und ökonomische Nachhaltigkeit

- Maintainability: technische, soziale und ökonomische Nachhaltigkeit 
- Efficiency: technische, ökologische und ökonomische Nachhaltigkeit

- Usability: soziale und ökonomische Nachhaltigkeit

- Trust: technische und soziale Nachhaltigkeit

- Usefulness: technische und soziale Nachhaltigkeit

- Recoverability: technische Nachhaltigkeit

Bei den Kriterien Trust, Usefulness und Recoverability herrscht noch Uneinigkeit, ob diese zu ökonomischer Nachhaltigkeit beitragen oder nicht (vgl. CondoriFernandez und Lago 2019; Fernandez et al. 2019; Condori-Fernandez et al. 2020).

Darüber hinaus haben die AutorInnen (Fernandez et al. 2019; Condori-Fernandez et al. 2020) das ISO/IEC 25010 um die Merkmale Robustness, Data Privacy, Timeliness, Regulation compliance, Scalability und Tailorability erweitert, welche im Zuge von industriellen Projekten als relevant angesehen wurden.

\section{Green und Sustainable Software Qualitätsattribute}

Im Zuge dieser Studie wurden insgesamt 7 Modelle ausgewählt, und in Kap. 4 detaillierter dargestellt, welche Green und Sustainable Software im Kontext von Softwarequalität adressieren. Im Folgenden wird dargestellt, wie diese 7 Modelle Nachhaltigkeit berücksichtigen, welche ISO/IEC-Kriterien als relevant für die Entwicklung von Green und Sustainable Software angesehen werden und wie das jeweilige Modell zum ISO/IEC 25010 in Beziehung steht.

Im Hinblick auf Nachhaltigkeit adressieren 6 von 7 Modellen (mit Ausnahme von Modell 6) die Dimension der ökologischen Nachhaltigkeit. Auf Rang 2 befindet sich soziale Nachhaltigkeit, welche von Modell 1, 3 und 7 aufgegriffen wird. Ökonomische sowie technische Nachhaltigkeit befinden sich auf Rang 3 und werden jeweils von den Modellen 3 und 7 adressiert.

Tab. 2 Grüne und nachhaltige Produktqualitätskriterien

\begin{tabular}{lll}
\hline $\begin{array}{l}\text { Product Quality Model (PQ) } \\
\text { Kriterium }\end{array}$ & Subkriterium & Häufigkeit \\
\hline Performance efficiency & Resource utilization & $4 / 7$ \\
& & $3 / 7$ \\
Usability & Accessibility & $5 / 7$ \\
& & $3 / 7$ \\
Reliability & & $3 / 7$ \\
Functional suitability & & $3 / 7$ \\
Portability & Adaptability & $3 / 7$ \\
& & $2 / 7$ \\
Maintainability & Reusability & $2 / 7$ \\
& Modifiability & $2 / 7$ \\
& & $2 / 7$ \\
\hline
\end{tabular}


Im Spannungsfeld von Green und Sustainable Software und Softwarequalität werden die in Tab. 2 ersichtlichen Kriterien des ISO/IEC 25010 öfter als einmal genannt und daher von uns zumindest als relevant betrachtet.

Tab. 2 zeigt den breiten Zugang zum Thema Green und Sustainable Software auf. Das Thema Performance Efficiency hat erwartungsgemäß eine hohe Bedeutung, der inhaltliche Zusammenhang zum Thema ökologische Nachhaltigkeit und damit Green Software erschließt sich dabei unmittelbar. Die hohe Bedeutung von Usability und Functional Suitability ist vor dem Hintergrund der sozial nachhaltigen und langfristigen Nutzung von Software durch unterschiedlichste Benutzergruppen sehr gut verständlich und daher aus unserer Sicht auch wichtig. Die identifizierten Qualitätsattribute Reliability, Portability und Maintainability repräsentieren jedenfalls die ökonomische und technische Nachhaltigkeit auf die Produktqualität eines Softwareproduktes und sollten daher auch immer im Auge behalten werden.

\section{Zusammenfassung, Diskussion und Ausblick}

Die im ISO/IEC-Standard 25010 (ISO/IEC 25010 2011) enthaltenen Modelle zur Produktqualität (Product Quality, PQ) und zur Nutzungsqualität (Quality in Use, $Q i U)$ bildeten die Basis für die im Rahmen dieser Publikation durchgeführte Analyse. Insgesamt wurden 7 Modelle gefunden, welche Softwarequalität im Zusammenhang mit Nachhaltigkeit betrachten. Einteilen lassen sich diese Modelle einerseits nach ihrer Beziehung zum ISO/IEC 25010 und andererseits danach, wie sie nachhaltige Kriterien und Dimensionen berücksichtigen.

Einen direkten Bezug zum ISO-Standard stellen die Modelle $25010+S$ von $\mathrm{Ca}-$ lero et al. (2015), das Green/internal external quality model von Beghoura et al. (2017) und der Ansatz von Koçak et al. (2015) her. Der Beitrag dieser AutorInnen liegt dabei in der Ergänzung des ISO/IEC 25010 um eine ökologische Nachhaltigkeitsdimension. Calero et al. haben diese als Greenability bezeichnet. Bei Beghoura et al. heißt die Dimension Green Efficiency und bei Koçak et al. Environmental.

Die Autorinnen Condori-Fernandez und Lago (2018b) nehmen zum einen eine Zuordnung der ISO-Qualitätskriterien zu den Dimensionen sozialer, technischer, ökologischer und ökonomischer Nachhaltigkeit vor, zum anderen erweitern sie das ISO/IEC 25010 um die Merkmale Robustness, Data Privacy, Timeliness, Regulation compliance, Scalability und Tailorability.

Der Beitrag von Gordieiev und Kharchenko (2018) liegt in der Bewertung von PQ-Kriterien nach deren Relevanz für Green IT. Konkret haben die Autoren Performance Efficiency, Functional Suitability, Reliability und Usabillity als relevant erachtet.

Einen indirekten Bezug zum ISO/IEC 25010 stellen Kern et al. (2013) sowie Amri und Bellamine Ben Saoud (2014b) her. Beiden Modellen ist gemein, dass sie Nachhaltigkeit als eigenes Softwarequalitätskonzept ansehen. Kern et al. (2013) haben das dreidimensionale Quality Model for Green and Sustainable Software veröffentlicht, um Effekte erster, zweiter und dritter Ordnung bei der Entwicklung von Software zu berücksichtigen. Sie adressieren in ihrem Modell soziale und ökologi- 
sche Nachhaltigkeit und erachten die folgenden Kriterien des ISO/IEC 25010 als relevant für eine nachhaltige Softwareentwicklung: Efficiency, Reusability, Modifiability und Usability.

Amri und Bellamine Ben Saoud (2014b) entwickelten das Generic Sustainable Software Star Model (GS3M) und adressieren mit jeder Sternzacke eine der folgenden Dimensionen: Umwelt, Wirtschaft, Soziales, Individuelles und Technisches. Sie nehmen auf die Norm ISO/IEC 25010 (2011) insoweit Bezug, als dass ihre generalisierten Attribute durch Qualitätskriterien aus der Norm konkretisiert werden können. Als Beispiel führen sie Accessibility an, welchem sie einen sozialen Mehrwert für AnwenderInnen eines Softwareprodukts attestieren. In Form von Green Software beziehen sie sich auf die ISO-Kriterien Performance Efficiency und indirekt auch auf Resource utilization. Als Voraussetzungen für die Dauerhaftigkeit von Software sehen Amri und Bellamine Ben Saoud die Kriterien Maintainability, Portability und Usability an.

Zusammenfassend lässt sich sagen, dass im Kontext von Green und Sustainable Software die folgenden Kriterien des ISO/IEC 25010 am häufigsten genannt wurden: Usability, Performance Efficiency, Functional Suitability, Resource utilization, Reliability und Portability. Die AutorInnen Calero et al. (2015) sowie Condori-Fernandez und Lago (2018b) haben in ihren Publikationen zusätzlich das QiU-Modell betrachtet und Freedom from risk als besonders relevant für die Entwicklung von Green und Sustainable Software angesehen. Für PraktikerInnen bedeutet das, dass das bestehende ISO/IEC 25010 Modell durchaus geeignet ist, das Thema Green und Sustainable Software auf Basis dieser Qualitätsattribute zumindest teilweise zu messen. Dies bedeutet auch, dass bereits Metriken für diese Qualitätsattribute vorhanden sind, welche auch für die quantitative Messung der Nachhaltigkeit von Software verwendet werden können.

Unabhängig davon kommen wir zu dem Ergebnis, dass das Feld rund um Green und Sustainable Software nach wie vor heterogen ist und noch kein umfassender Konsens herrscht. Es besteht jedoch immerhin Einigkeit in Bezug auf einige Qualitätsattribute (z. B. Ressource utilization und Portability) und dass diese wesentlich dafür sind, die Aspekte Green und Sustainable Software systematisch zu erfassen. Wir planen, auf Basis des Ansatzes von Calero et al. (2015), ein operatives Qualitätsmodell für Green und Sustainable Software zu entwickeln, das die objektive Messung der Greenability einer Software ermöglicht.

Funding Open access funding provided by Johannes Kepler University Linz.

Open Access Dieser Artikel wird unter der Creative Commons Namensnennung 4.0 International Lizenz veröffentlicht, welche die Nutzung, Vervielfältigung, Bearbeitung, Verbreitung und Wiedergabe in jeglichem Medium und Format erlaubt, sofern Sie den/die ursprünglichen Autor(en) und die Quelle ordnungsgemäß nennen, einen Link zur Creative Commons Lizenz beifügen und angeben, ob Änderungen vorgenommen wurden.

Die in diesem Artikel enthaltenen Bilder und sonstiges Drittmaterial unterliegen ebenfalls der genannten Creative Commons Lizenz, sofern sich aus der Abbildungslegende nichts anderes ergibt. Sofern das betreffende Material nicht unter der genannten Creative Commons Lizenz steht und die betreffende Handlung nicht nach gesetzlichen Vorschriften erlaubt ist, ist für die oben aufgeführten Weiterverwendungen des Materials die Einwilligung des jeweiligen Rechteinhabers einzuholen. 
Weitere Details zur Lizenz entnehmen Sie bitte der Lizenzinformation auf http://creativecommons.org/ licenses/by/4.0/deed.de.

\section{Literatur}

Amri R, Ben Saoud BN (2014a) Using sustainable software model to identify constraints and opportunities toward sustainability in software engineering. Vision 2020: Sustainable Growth, Economic Development, and Global Competitiveness-Proceedings of the 23rd International Business Information Management Association Conference, IBIMA 2014. Bd. 1, S 1600-1607

Amri R, Ben Saoud BN (2014b) Towards a generic sustainable software model. 2014b Fourth International Conference on Advances in Computing and Communications, S 231-234 https://doi.org/10.1109/ ICACC.2014.62

Beghoura MA, Boubetra A, Boukerram A (2017) Green software requirements and measurement: random decision forests-based software energy consumption profiling. Requir Eng 22(1):27-40. https://doi. org/10.1007/s00766-015-0234-2

Bundeskanzleramt Österreich Nachhaltige Entwicklung - Agenda 2030/SDGs. https://www. bundeskanzleramt.gv.at/themen/nachhaltige-entwicklung-agenda-2030.html. Zugegriffen: 8. Dez. 2020

Calero C, Bertoa MF, Moraga MÁ (2013a) A systematic literature review for software sustainability measures. (2013a) 2nd International Workshop on Green and Sustainable Software (GREENS), S 46-53 https://doi.org/10.1109/GREENS.2013.6606421

Calero C, Bertoa MF, Moraga MÁ (2013b) Sustainability and quality: icing on the cake. Proceedings of the 2nd International Workshop on Requirements Engineering for Sustainable Systems. Bd. 995, S 4

Calero C, Mancebo J, García F, Moraga MÁ, Berná JAG, Fernández-Alemán JL, Toval A (2020) 5Ws of green and sustainable software. Tinshhua Sci Technol 25(3):401-414. https://doi.org/10.26599/TST. 2019.9010006

Calero C, Moraga M, Bertoa MF, Duboc L (2014a) Quality in use and software greenability. RE4SuSy@RE

Calero C, Moraga MA, Bertoa MF (2014b) Towards a software product sustainability model. arxiv:1309.1640. http://arxiv.org/abs/1309.1640. Zugegriffen: 15. Okt. 2020

Calero C, Moraga MÁ, Bertoa MF, Duboc L (2015) Green software and software quality. Green Softw Eng. https://doi.org/10.1007/978-3-319-08581-4_10

Condori-Fernandez N, Lago P (2018a) Characterizing the contribution of quality requirements to software sustainability. J Syst Softw 137:289-305. https://doi.org/10.1016/j.jss.2017.12.005

Condori-Fernandez N, Lago P (2018b) A software sustainability-quality model. https://research.vu.nl/en/ publications/a-sustainability-quality-model-version-10. Zugegriffen: 18. Nov. 2020

Condori-Fernandez N, Lago P (2019) Towards a software sustainability-quality model: insights from a multi-case study. 2019 13th International Conference on Research Challenges in Information Science (RCIS), S 1-11 https://doi.org/10.1109/RCIS.2019.8877084

Condori-Fernandez N, Lago P, Luaces MR, Places ÁS (2020) An action research for improving the sustainability assessment framework instruments. Sustainability 12(4):1682. https://doi.org/10.3390/ su12041682

Fernandez NC, Lago P, Luaces MR, Places ÁS, Folgueira LG (2019) Using participatory technical-action-research to validate a software sustainability model. CEUR Workshop Proceedings, 2382. www. scopus.com. Zugegriffen: 30. Dez. 2020

García-Mireles GA, Moraga MÁ, García F, Calero C, Piattini M (2018) Interactions between environmental sustainability goals and software product quality: a mapping study. Inf Softw Technol 95:108-129. https://doi.org/10.1016/j.infsof.2017.10.002

Gordieiev O, Kharchenko V (2018) IT-oriented software quality models and evolution of the prevailing characteristics. 2018 IEEE 9th International Conference on Dependable Systems, Services and Technologies (DESSERT), S 375-380 https://doi.org/10.1109/DESSERT.2018.8409162

Gordieiev O, Kharchenko V, Fusani M (2015) Evolution of software quality models: green and reliability issues. CEUR Workshop Proceedings, 1356, S 432-445

Gordieiev O, Kharchenko V, Fusani M (2016) Software quality standards and models evolution: greenness and reliability issues. In: Yakovyna V, Mayr HC, Nikitchenko M, Zholtkevych G, Spivakovsky A, Batsakis S (Hrsg) Information and communication technologies in education, research, and industrial applications. Springer, Berlin Heidelberg, S 38-55 https://doi.org/10.1007/978-3-319-30246-1_3 
ISO ISO/IEC 9126-1:2001. https://www.iso.org/cms/render/live/en/sites/isoorg/contents/data/standard/02/ 27/22749.html. Zugegriffen: 19. Jan. 2021

ISO ISO/IEC CD 25010. https://www.iso.org/cms/render/live/en/sites/isoorg/contents/data/standard/07/ 81/78176.html. Zugegriffen: 08. Jan. 2021

ISO/IEC 25010:2011 (2011) Systems and software engineering-Systems and software Quality Requirements and Evaluation (SQuaRE)—System and software quality models. https://doi.org/10.3403/ 30215101

Kern E, Dick M, Naumann S, Guldner A, Johann T (2013) Green software and green software engineering-Definitions, measurements, and quality aspects. First International Conference on Information and Communication for Sustainability. ICT4S 2013 Proceedings

Kläs M, Heidrich J, Münch J, Trendowicz A (2009) CQML scheme: a classification scheme for comprehensive quality model landscapes. In: 35th Euromicro conference on software engineering and advanced applications (SEAA). IEEE, Patras, S 243-250 https://doi.org/10.1109/SEAA.2009.88

Koçak SA, Gonzales Calienes G, Alptekin GI, Bener AB (2013) Requirements prioritization framework for developing green and sustainable software using ANP-based decision making. In: Page B, Fleischer AG, Göbel J, Wohlgemuth V (Hrsg) Proceedings of the 27th conference on environmental informatics-informatics for environmental protection, sustainable development and risk management. Shaker,

Koçak SA, Alptekin GI, Bener AB (2014) Evaluation of software product quality attributes and environmental attributes using ANP decision framework. RE4SuSy@RE, S 8

Koçak SA, Alptekin GI, Bener AB (2015) Integrating environmental sustainability in software product quality, S 8

Naumann S, Dick M, Kern E, Johann T (2011) The GREENSOFT model: a reference model for green and sustainable software and its engineering. Sustain Comput Inform Syst 1(4):294-304. https://doi.org/ 10.1016/j.suscom.2011.06.004

Naumann S, Kern E, Dick M, Johann T (2015) Sustainable software engineering: process and quality models, life cycle, and social aspects. In: Hilty LM, Aebischer B (Hrsg) ICT innovations for sustainability, Bd. 310. Springer, Berlin Heidelberg, S 191-205 https://doi.org/10.1007/978-3-319-092287_11

Penzenstadler B, Femmer H (2013) A generic model for sustainability with process- and product-specific instances. Proceedings of the 2013 Workshop on Green in/by Software Engineering-GIBSE '13, S 3 https://doi.org/10.1145/2451605.2451609

SWEBOK. https://www.ieee.org/about/ieee-india/ieee-computer-society-india/swebok.html. Zugegriffen: 06. Jan. 2021

United Nations Sustainable Development Sustainable development goals. https://www.un.org/ sustainabledevelopment/. Zugegriffen: 6. Jan. 2021 